\title{
Traduction et linguistique diachronique: une relation de pourvoyeur à bénéficiaire
}

\author{
Michèle Goyens \& Willy Van Hoecke \\ Katholieke Universiteit Leuven
}

Translations, which are frequently used in contrastive linguistics to analyse syntactic or semantic features in different languages, are also precious instruments for the diachronic study of linguistic phenomena, especially if the corpus texts imply several historical stages of one language. These successive translations are all authentic testimonies by native speakers to the most appropriate expression of one and the same message in their language system.

In this paper, we present the methodology underlying our approach and describe the basic corpus. Next, we sketch the current state of (the) research which is being carried out in this respect at the Catholic University of Leuven (K.U. Leuven). Recently, two new projects have enabled us to enlarge the corpus texts and to give a new orientation to our approach. We will therefore outline the prospects of our research.

\section{Introduction}

En linguistique contrastive, les traductions sont fréquemment mises à profit de nos jours pour étudier des phénomènes syntaxiques ou sémantiques dans différentes langues et pour montrer ainsi leur fonctionnement parallèle ou au contraire divergent. Par sa nature fondamentalement empirique, cette approche donne en même temps à la recherche linguistique une orientation "réaliste" qui permet de remédier aux risques d'une introspection trop poussée.

Pour l'étude de l'évolution d'une langue, les traductions s'avèrent également être de précieuses sources, certainement si un texte ancien est traduit dans un idiome issu de la langue dans laquelle ce texte a été composé. Depuis bientôt vingt ans, nous travaillons à l'Université catholique de Louvain (K.U. Leuven) à un projet de recherche que nous avons baptisé Traduction et Changement Linguistique. L'idée qui sous-tend le projet est que, lorsqu' on dispose d'un texte source et d'un certain nombre de traductions "fidèles", on peut les considérer comme autant de "réalisations" adéquates d'un même message par différents "locuteurs natifs", chacun dans sa langue maternelle propre.

Dans cette contribution, nous aimerions d'abord préciser notre méthode et présenter le corpus de base que nous utilisons (1.). Ensuite, nous voudrions esquisser les recherches que nous avons lancées depuis bientôt vingt ans à la K.U. Leuven dans cette optique et présenter certains résultats qui nous semblent particulièrement pertinents (2.). Récemment, le corpus a 
connu un élargissement, grâce à deux nouveaux projets de recherche qui nous permettent de développer de nouvelles approches dans l'évolution de la langue (3.). Ce bref aperçu se terminera par l'esquisse d'une série de perspectives qu'offre notre méthode de travail (4.).

\section{Corpus et méthodologie}

Comme nous venons de dire, l'idée qui sous-tend notre projet est que, lorsqu'on dispose d'un texte source et d'un certain nombre de traductions "fidèles", on peut admettre que le texte source et ses traductions forment autant de témoignages authentiques de locuteurs natifs sur l'expression la plus appropriée d'un même message dans leur système linguistique. Dans le cas de traductions réalisées dans des langues non apparentées, l'analyse fera apparaître certaines différences distinctives et d'éventuelles ressemblances et apportera ainsi des éléments empiriques à la linguistique contrastive et à la typologie linguistique.

De même, la méthode peut être particulièrement utile à la linguistique diachronique: si un texte ancien est traduit dans un idiome issu de la langue dans laquelle ce texte a été composé, la traduction permet de saisir sur le vif des changements qui ont transformé la langue mère et provoqué l'émergence d'un nouveau système linguistique. C'est surtout dans cette dernière optique que nous avons travaillé jusqu'ici.

Dans le concret, nous nous servons de deux traités de rhétorique latins de l'époque classique, à savoir le De Inventione de Cicéron et la Rhetorica ad Herennium. Il s'agit de deux textes assez scolaires qui sont écrits en une langue peu recherchée, proche sans doute du latin utilisé couramment dans les écoles de l'époque. Dans le cadre plutôt rigide imposé par le sujet, les deux œuvres évoquent des aspects assez variés de la vie romaine contemporaine, allant de questions philosophiques et littéraires à des problèmes de procédure juridique ou d'organisation politique et militaire. On y trouve donc un vocabulaire relativement riche, mais peu de subtilités syntaxiques ou de finesses stylistiques: somme toute, un texte source qui n'est pas de nature à inciter les traducteurs à des stratégies d'adaptation particulières. Nous avons saisi le texte de ces traités tel qu'il a été établi par Stroebel (éd. 1965) et Marx (éd. 1964) pour la collection Bibliotheca scriptorum Graecorum et Romanorum Teubneriana et par Achard (éd. 1994) et Achard (éd. 1989) pour la Collection des Universités de France.

À partir du $12^{\mathrm{e}}$ siècle, ces deux textes ont été rassemblés dans un manuel de base destiné à l'enseignement médiéval de la rhétorique: on croyait, en effet, que le De Inventione, qui ne traite que de la première partie de la rhétorique, à savoir l'inventio, était resté inachevé et que Cicéron l'avait complété dans la Rhetorica ad Herennium qu'on attribuait à tort également à lui. En 1282, un certain Jean d'Antioche a traduit l'ensemble en ancien français sous le titre de Rettorique de Marc Tulles Cyceron. Le texte est divisé en 206 chapitres: il commence par un prologue, suivi de la traduction proprement dite comprenant les chapitres 2-75 et 76-204, qui correspondent 
respectivement aux deux livres du De Inventione et aux quatre livres de la Rhetorica ad Herennium; le tout se termine par une postface et un épilogue.

La traduction est conservée dans un seul manuscrit, à savoir le ms. Chantilly, Musée Condé 483. Ce codex assez luxueux a été signalé et décrit en détail par Delisle (1899), qui a avancé plusieurs arguments pour le considérer comme un manuscrit original. La traduction a été réalisée à Acre en Terre Sainte, à la demande de Guillaume de Saint-Etienne, un frère de l'Hôpital de Jérusalem. Celui-ci le considérait manifestement comme une des grandes sommes antiques non seulement en matière de rhétorique, mais aussi et surtout dans le domaine de la philosophie et du droit, car il s'en est servi dans une longue digression philosophique, intitulée Livre Satérian, qu'il a insérée dans une vaste compilation sur l'ordre de l'Hôpital, rédigée lorsqu'il était devenu commandeur de l'ordre dans l'île de Chypre. Selon la dernière phrase de l'épilogue, elle aurait été terminée au mois de septembre 1296 (Delisle 1906). Conservée dans le seul ms. PARIS, B.N.F., fr. 6049, elle est restée inédite.

La traduction de Jean d'Antioche est assez exceptionnelle, car dans sa Postface le traducteur déclare explicitement qu'il a voulu respecter la maniere dou tracter de l'auctor a son pooir et au plus pres qu'il pot. Toutefois il n' a pas pu porsivre l'auctor en la maniere dou parler, car en bon translatour il a dû se conformer aux propriétés de la langue d'arrivée, l'ancien français. Or il est clair pour lui que la maniere dou parler en latin n'est pas semblable generaument a cele dou françois et que de nombreuses différences entre les deux langues compliquent sérieusement la tâche du traducteur. Jean d'Antioche a pu translater aucune fois parole por parole, et aucune fois et plus sovent sentence por sentence, mais aucune fois, por la grant oscurté de la sentence, li covint il sozjoindre et acreistre. D'ailleurs, si d'aucuns pensent trouver des fautes dans sa version, Jean d'Antioche leur demande de bien comparer les deus letres dou latin et dou françois pour voir si vraiment l'en le peut meauz faire si on veut à la fois respecter le texte original et les particularités de la langue cible. Nous avons affaire à une traduction assez remarquable, faite par un clerc qui s'est livré à "un effort d'exactitude et de style qui ne s'était pas encore manifesté" (Monfrin 1964:224) et qui avait en plus "une vision assez unique et originale du rapport du français au latin” (Lusignan 1986:145).

La traduction a été éditée par quatorze étudiants sous forme de mémoires de licence, dirigés par Willy Van Hoecke et présentés à la section de philologie romane de la K.U. Leuven entre 1972 et 1984 . Cette édition provisoire a été uniformisée, collationnée sur le manuscrit et numérisée. Le texte est actuellement disponible dans une version informatisée destinée aux recherches conçues dans le cadre de notre projet, ainsi que sous la forme qu'il prendra dans l'édition définitive (Van Hoecke éd. s.d.).

A notre connaissance, les deux traités n'ont plus été traduits en français avant la fin du $16^{\mathrm{e}}$ siècle, mais depuis une dizaine de versions ont paru. Pour nos comparaisons, nous avons surtout utilisé jusqu'ici les traductions récentes de Bornecque (éd. 1932a, éd. 1932b) et celles qui accompagnent les éditions déjà citées d'Achard (éd. 1989, éd. 1994) 
En comparant minutieusement les passages du texte source et les transpositions de ceux-ci par les différents traducteurs français, il est possible de détecter sur le vif certains changements que la langue a connus. Nous avons décrit ailleurs (Goyens \& Van Hoecke 1992) comment nous procédons dans le concret et insisté sur les problèmes qui gênent inévitablement toute méthode axée sur des corpus. Inutile de dire que la collation des témoins est un travail aride et de longue haleine, qui réclame une application constante et soutenue et qui se révèle plus d'une fois être stérile. Par exemple, dans certains passages, relativement rares heureusement, la comparaison est impossible parce que les traductions s'écartent manifestement du texte latin tel qu'il a été établi par les éditeurs modernes. Parfois la comparaison se complique parce que les traducteurs rendent le texte latin à l'aide d'une construction assez différente de celle de leur modèle. Mais en moyenne, le taux de stérilité du corpus n'atteint généralement pas $20 \%$.

\section{Résultats}

Les études que nous avons réalisées jusqu'à présent, dont nous avons présenté les principaux résultats au colloque La Recherche: Bilan et Perspectives organisé par Giuseppe Di Stefano à Montréal en octobre 1998 (Goyens \& Van Hoecke 2000), nous semblent assez prometteuses. En effet, pour plus d'une question, l'analyse offre des éléments de comparaison authentiques assez précieux. Certes, elle n'ouvre guère des perspectives entièrement nouvelles, mais permet néanmoins de préciser et de nuancer certains aspects des descriptions existantes. En outre, en tant que recherche basée sur un corpus, elle donne à celles-ci une orientation quantitative.

Ces analyses se concentrent principalement sur le lexique et la morphosyntaxe. Dans le premier domaine, l'évolution de la terminologie juridique ainsi que de lexèmes isolés tels que RES, CAUSA, UIS, HOMO, TEMPUS, LOCUS, a fait l'objet d'une série d'études ${ }^{1}$, dont l'intérêt réside essentiellement dans la mise à nu de glissements sémantiques de la forme latine de départ. Prenons en guise d'exemple le substantif latin GENUs, "espèce, sorte, genre", qui évolue en français ancien vers la forme populaire giens et les formes savantes gendre, genre ou gerre. Or, notre corpus révèle que le traducteur médiéval, Jean d'Antioche, recourt le plus souvent au syntagme nominal general maniere, composé du substantif maniere et de l'adjectif general, pour rendre le sens que prend GENUS dans sa source latine. Le traducteur moderne, par contre, se sert systématiquement de la forme savante genre pour rendre le terme. L'analyse nous permet donc de saisir sur le vif l'emploi concret d'un lexème par le traducteur qui doit faire un choix pour rendre le mieux possible le sens des éléments qu'il trouve dans son texte source. En ce qui concerne l'ancien français, nous voyons dans cet exemple concret que le traducteur n'opte ni pour le terme populaire existant giens, ni pour une forme savante déjà attestée, mais préfère recourir à une combinaison de deux lexèmes qui selon lui est plus adéquate.

Dans le domaine de la morphosyntaxe, c'est la composition du syn- 
tagme nominal et le développement du système de la détermination nominale qui ont été analysés en premier lieu (voir notamment Goyens 1994). Ici, la comparaison de la traduction médiévale avec les textes sources latins et les traductions modernes fait clairement apparaître la position intermédiaire de cette période, avec des données chiffrées à l'appui: alors que le latin ne connaît pas de système référentiel explicite, les contextes correspondants dans la version médiévale révèlent clairement le besoin d'explicitation et de précision de la référence, situation qui devient encore plus manifeste dans les traductions modernes. Concrètement, l'absence d'article devant le nom, que nous relevons dans $35,9 \%$ des syntagmes nominaux de la traduction en ancien français ${ }^{2}$, se restreint pour n'apparaître plus que dans $14,5 \%$ des syntagmes nominaux de la traduction moderne. Cette diminution va de pair avec l'emploi de plus en plus fréquent de l'article défini, qui passe de 43,4\% dans la traduction médiévale, à 54,9\% dans la traduction moderne, mais surtout de l'article indéfini, qui marque une montée spectaculaire de 2,3\% dans la traduction médiévale à $11,2 \%$ dans la traduction moderne. Si des études préalables avaient déjà relevé ce développement du système de détermination français, elles n'avaient pas pu mettre le doigt sur la différence assez remarquable dans le développement de l'emploi de l'article indéfini face au défini. En effet, l'article indéfini, avec un sens numéral encore très net dans la version en ancien français, développe des emplois par opposition avec le défini et s'intègre ainsi bien nettement dans le système des articles ${ }^{3}$.

La phrase simple ou complexe a également été soumise à l'étude à partir de notre corpus. Citons par exemple l'analyse du système de l'interrogation et celle de l'évolution de divers connecteurs. ${ }^{4}$ L'utilisation du corpus de traductions permet d'apporter d'importantes retouches aux descriptions existantes, qui sont souvent trop simplificatrices.

En tant que recherche basée sur un corpus, elle donne aux analyses une orientation quantitative: alors que les descriptions existantes mettent souvent sur un pied d'égalité des phénomènes dont la fréquence d'apparition peut varier nettement, les études basées sur des corpus de traductions permettent de montrer avec une grande précision l'impact de certaines évolutions, ou leur caractère plus ou moins significatif du point de vue de leur fréquence d'apparition, comme c'était le cas par exemple pour l'émergence de l'article indéfini $u n$, dont on n'avait pas encore pu apprécier de façon si précise ni le développement quantitatif, ni l'évolution sémantique.

\section{Elargissement du corpus}

Non seulement nous comptons continuer à explorer le corpus déjà constitué, mais en outre nous travaillons à son élargissement. En effet, actuellement, deux nouveaux projets contribuent à l'extension de notre corpus de traductions, extension qui ne fait pas seulement augmenter le nombre de textes, mais qui permet aussi de développer de nouvelles approches dans l'étude de l'évolution du français, en associant cette fois des traductions du grec au latin à celles du latin au français (ancien et moderne). 
Un premier projet, pour lequel nous pouvons bénéficier des subsides du Fonds de recherche de la K.U.Leuven 5 , est consacré au développement du vocabulaire biologique au moyen âge et se divise en deux volets, reflétant deux corpus parallèles, à savoir la traduction du De Historia Animalium d'Aristote en latin par Guillaume de Moerbeke d'une part ${ }^{6}$, et diverses traductions anonymes du texte latin De Falconibus d'Albert le Grand en moyen français ( $\mathrm{du} 14^{\mathrm{e}}$ au $16^{\mathrm{e}}$ siècle) d'autre part. Ce projet se réalise en collaboration avec nos collègues du centre de recherche de l'Aristoteles Latinus ${ }^{7}$, qui s'occupent du premier volet de l'étude. Pour le second, nous pouvons compter sur la coopération d'An Smets, qui termine l'édition des quatre traductions en moyen français du De Falconibus, et étudie le développement du vocabulaire scientifique relatif à la fauconnerie ${ }^{8}$.

Par rapport au corpus existant, le corpus développé ici présente au moins deux particularités. Ainsi, les traducteurs en jeu se trouvent dans des situations différentes: celui qui traduit du grec au latin, le latin médiéval, utilise une langue savante, qu'il a apprise dans une situation scolaire; celui qui traduit en (moyen) français à partir du latin médiéval, une langue savante, utilise sa langue maternelle, qu'il connaît depuis toujours et qu'il pratique chaque jour, dans des circonstances divergentes. Les deux types de traducteurs essaient toutefois de rendre le message de la façon la plus adéquate possible.

Ce corpus peut être utilisé en première instance pour explorer des aspects concernant le latin médiéval et le moyen français. En effet, aux deux niveaux mentionnés, le traducteur doit tout d'abord faire une série de choix pour sélectionner les équivalents les plus adéquats pour sa traduction. A cet effet, il pouvait recourir à diverses stratégies: chercher un équivalent dans l'usage contemporain de sa langue cible, ou s'inspirer de sources existantes, disponibles sous forme de traductions ou rédigées dans d'autres langues. Si toutefois sa recherche s'avérait stérile, il devait lui-même proposer un équivalent, qu'il pouvait trouver en calquant sa source, ou en utilisant un terme existant auquel il donnait un sens légèrement déviant. Le fait que nous disposons de diverses traductions en moyen français du De Falconibus permet d'ailleurs des comparaisons intéressantes au sein d'une même langue.

Si un tel corpus permet tout d'abord des études sur le latin médiéval d'une part, et sur le moyen français de l'autre, il est particulièrement intéressant quant à l'analyse des stratégies de traduction développées et utilisées par les différents traducteurs, ayant chacun ses propres objectifs et un public particulier. Le premier écrit un traité savant, destiné à un public savant, tandis que le second veut se faire comprendre à un public qui n'est pas forcément lettré! Il est donc important de replacer dans son contexte socio-culturel l'acte de traduire afin d'en dégager les stratégies spécifiques.

Un autre projet, qui a été entamé simultanément au précédent par Pieter De Leemans et Michèle Goyens, implique un corpus analogue, mais dont les différentes composantes sont encore mieux liées. Il comprend le traité connu sous le nom de Problèmes (Problemata), composé à l'origine 
par Aristote lui-même et plus tard complété par ses successeurs (d'où aussi le nom de Pseudo-Aristote). Le texte est une collection pittoresque d'exposés sur des thèmes très divers, tels que la médecine, l'harmonie, la météorologie, les émotions, mais aussi les animaux, les pierres et les plantes, permettant de satisfaire la curiosité de l'homme scientifique médiéval. ${ }^{9}$ Vers 1260, ce traité a été traduit en latin par Barthélémy de Messine. Un demi-siècle plus tard, Pierre d'Abano ajouta un commentaire à cette traduction. En se basant sur ces deux écrits, un certain Evrart de Conty réalisa, vers 1380, une traduction en français des Problèmes, à la demande de Charles V le Sage, qui était connu pour son grand amour des lettres et les nombreux savants et traducteurs dont il s'était entouré ${ }^{10}$.

La traduction d'Evrart est conçue de la façon suivante. Pour chaque problème, il traduit d'abord de manière assez fidèle le texte de Barthélémy; ensuite, il traduit le commentaire de Pierre d'Abano, auquel il ajoute fréquemment des développements personnels, ce qui rend cette partie moins transparente et donc plus complexe à analyser.

Ce corpus est particulièrement intéressant pour l'analyse du vocabulaire scientifique. En effet, le texte, très long, compte 38 sections de longueur inégale, divisées en une série de problèmes: comme nous l'avons déjà montré, ces sections embrassent des thèmes scientifiques mais aussi artistiques fort divergents.

Nous avons jusqu'à présent réalisé quelques études concernant le vocabulaire utilisé par les différents traducteurs et par le commentateur ${ }^{11}$, et celles-ci révèlent une tradition fort différente dans les méthodes de traduction utilisées par Barthélémy de Messine et Evrart de Conty. Ainsi, Barthélémy traduit mot à mot sa source grecque, et emprunte régulièrement des termes à celle-ci, que le commentateur précise ou corrige même souvent. Quant à Evrart de Conty, il traduit plutôt ad sensum, jugeant sa langue en général trop éloignée du modèle latin pour suivre celui-ci de très près; il est bien conscient d'ailleurs qu'il traduit non un texte original, mais déjà une traduction qu'il qualifie souvent de "corrompue". Concrètement, en traduisant il utilise tantôt des termes existants, qu'il a sans doute trouvés dans des ouvrages qu'il avait à sa disposition, tantôt il semble recourir à des néologismes, qui ont le plus souvent survécu en français. Ces données nous permettent à plusieurs reprises de corriger les informations historiques fournies par les dictionnaires.

Illustrons ces propos par deux exemples. Dans le problème consacré à la mélancolie (section XXX des Problèmes), le texte d'Aristote présente le terme "melankholikos" afin de rendre la notion de "être atteint par la mélancolie", la maladie causée par un excès de bile noire. La traduction latine ainsi que le commentaire rendent cette notion à l'aide de l'adjectif melancholicus, forme empruntée en latin classique déjà au grec (cf. OLD 1092). Evrart de Conty, quant à lui, utilise systématiquement l'adjectif melancolieus, et non melancolique, formes toutes deux attestées en ancien français (cf. TL V: 1356, 1358; GOD V: 221, 222 et X: 137) et pour lesquelles les dictionnaires ne mentionnent guère de différence sémantique. Or, Evrart utilise manifestement le terme melancolieus conformément à son étymologie. En effet, 
la forme melancolieus remonte à une forme au suffixe -OSUS, signifiant "plein de" (cf. Kühner-Holzweissig ${ }^{2} 1912: I, \S 225,8$ ), d'où le sens de "qui a un excès de bile noire", et donc, dans notre contexte, "qui est atteint par la maladie causée par la bile noire". L'adjectif melancolique par contre a pour origine une forme au suffixe -ICUS, qui signifie "relatif à, qui est propre à" (Rey dir. 1992: 1344), d'où le sens de "relatif à la bile noire", qui est beaucoup moins précis que dans le premier cas. Evrart est donc scrupuleux lorsqu'il choisit ses termes, et il tire un maximum de profit des formes attestées à son époque pour les spécialiser, conformément à leur étymologie, dans un emploi particulier.

Dans un autre cas, Evrart de Conty fait preuve d'innovation dans l'élaboration du vocabulaire scientifique. Ainsi, dans le sixième problème de la section XX traitant des plantes, il doit traduire le passage suivant, correspondant au texte latin de Barthélémy de Messine:

(1) Propter quid hec quidem incommestibilia sunt, hec vero commestibilia?

Pour rendre l'adjectif commestibilia, il utilise d'abord la périphrase bonnes a mengier:

(1a) Pour quoy est ce que aucunes des plantes et des choses qui naiscent en terre les aucunes sont aiables et bonnes a mengier, et les autres non? (Evrart de Conty, Problemes, XX, 6, fol. $\left.58 \mathrm{v}^{\circ}\right)^{12}$

Or, dès le moment où il traduit le commentaire de PA, Evrart de Conty utilise la forme comestibles, la première fois en réduplication synonymique avec bonnes a mengier, alors que le texte de Pierre d'Abano présente toujours commestibilia:

(2) La response de Aristote est fundee sur la difference des choses comestibles et bonnes a mengier a celles qui ne le sont mie. (Evrart de Conty, Problemes, XX, 6, fol. 59 r ${ }^{\circ}$ )

Dans la suite de la traduction, Evrart de Conty utilise systématiquement comestible, non redoublé cette fois. La forme comestible, remontant à COMESTUS (FEW II 940), est attestée selon les dictionnaires depuis la fin du $14 \mathrm{e}$ s., et plus précisément depuis Evrart de Conty ${ }^{13}$. Evrart est donc le premier à introduire la forme: elle lui permet de cerner par un lexème simple un sens particulier qui ne pouvait être rendu que par une périphrase, bon $a$ mengier.

Comme nous l'avons déjà mentionné, d'autres analyses nous ont permis de constater qu'Evrart de Conty enrichit régulièrement le vocabulaire scientifique français par des néologismes, enrichissement qui s'est réalisé grâce à l'acte de traduction. 


\section{Perspectives}

Nous espérons avoir montré le rôle extrêmement important que peuvent jouer les traductions dans l'étude de l'évolution d'une langue, et la richesse d'informations que celles-ci apportent et qui est due précisément au fait qu'elles doivent rendre des contenus sémantiques identiques par rapport à un modèle. C'est pourquoi le potentiel de ces corpus doit être mis à profit au maximum.

Un autre avantage de notre approche est son caractère empirique: le corpus permet d'extraire des données objectives et précises, que l'on peut soumettre dans certains cas à des analyses statistiques.

Nous envisageons dès lors de poursuivre les recherches entamées, tout d'abord dans les domaines que nous avons déjà explorés, à savoir le lexique et la morphosyntaxe. Il reste en effet des thèmes importants à explorer, comme la terminologie de la rhétorique mais aussi l'éclipse des cas latins et l'essor des prépositions, pour ne citer que deux exemples. Grâce à l'élargissement du corpus par les traductions parallèles, du grec au latin et du latin au français, de traités à orientation scientifique, nous avons l'occasion d'étudier systématiquement le développement du vocabulaire scientifique en latin et en français.

Quoi qu'il en soit, nous sommes d'avis que la linguistique diachronique trouve un intérêt indéniable dans la "fréquentation des textes de traduction", comme l'avait affirmé Charles Brucker (1977: 339), qui lui permettront de raffiner ses méthodes et techniques d'investigation.

\section{Bibliographie}

Achard, Guy (Ed.) (1989). Rhétorique à Herennius. Texte établi et traduit. Collection des Universités de France. Paris: Les Belles Lettres.

Achard, Guy (Ed.) (1994). Cicéron. De l'Invention. Texte établi et traduit. Collection des Universités de France. Paris: Les Belles Lettres.

Beullens, P. \& F. Bossier (Eds) (2000). De Historia Animalium. Translatio Guillelmi de Morbeka. Pars Prima: lib. I-V. Aristoteles Latinus XVII 2.I.1. LeidenBoston-Köln: Brill.

Bornecque, Henri (Ed.) (1932a). Cicéron. De l'Invention (De Inventione). Texte revu et traduit avec introduction et notes. Paris: Garnier.

Bornecque, Henri (Ed.) (1932b). Rhétorique à Herennius. Ouvrage longtemps attribué à Cicéron. Texte revu et traduit avec introduction et notes. Paris: Garnier.

Brucker, Charles (1977). "La valeur du témoignage linguistique des traductions médiévales: les constructions infinitives en moyen français." Danielle Buschinger (Ed.) (1977). Actes du colloque des 29 et 30 avril 1977. Linguistique et Philologie (application aux textes médiévaux) (Université de Picardie, Centre d'études médiévales). Paris: Champion, 325-344.

Carlier, Anne \& Michèle Goyens (1998). "De l'ancien français au français moderne: régression du degré zéro de détermination et restructuration du système des articles." Jean René Klein, Béatrice Lamiroy \& Jean-Marie Pierret (Eds) (1998). Théorie linguistique et applications informatiques. Actes du 16e 
Colloque européen sur la grammaire et le lexique comparés. Cahiers de l'Institut de Linguistique de Louvain 24, 77-112.

Delisle, Léopold (1899). "Notice sur la Rhétorique de Cicéron traduite par maître Jean d'Antioche, ms. 590 du Musée Condé." Notices et Extraits des manuscrits de la Bibliothèque Nationale et autres bibliothèques 36, 207-265.

Delisle, Léopold (1906). "Maître Jean d'Antioche, traducteur, et Frère Guillaume de Saint-Etienne, hospitalier". Histoire littéraire de la France 33, 1-40.

FEW: voir Wartburg (1928ss.).

GOD: voir Godefroy (1980-1902).

Godefroy, Fr. (1880-1902). Dictionnaire de l'ancienne langue française et de tous ses dialectes du IXe au XV siècle, composé d'après le dépouillement de tous les plus importants documents manuscrits ou imprimés qui se trouvent dans les grandes bibliothèques de la France et de l'Europe et dans les principales archives départementales, municipales, hospitalières ou privées. Paris: Vieweg.

Goyens, Michèle (1994). Emergence et évolution du syntagme nominal en français. Sciences pour la communication 43. Bern: P. Lang.

Goyens, Michèle (à par a). "Le développement du lexique scientifque français et la traduction des Problèmes d'Aristote par Evrart de Conty (ca 1380)." Thelema.

Goyens, Michèle (à par b). "Le lexique des plantes dans la traduction des Problèmes d'Aristote par Evrart de Conty (ca 1380)." Le Moyen Français.

Goyens, Michèle \& Pieter De Leemans (à par). "Traduire du grec au latin et du latin au français: un défi à la fidélité." Revue Romane.

Goyens, Michèle \& Willy Van Hoecke (1992). "La traduction comme témoin de l'évolution linguistique." Actas do XIX Congreso Internacional de Lingüística e Filoloxía Románicas. Universidade de Santiago de Compostela, 1989. Publicadas por Ramón Lorenzo. V. Gramática Histórica e Historia da Lingua. Coruña: Fundación "Pedro Barrié de la Maza, Conde de Fenosa", 13-32.

Goyens, Michèle \& Willy Van Hoecke (2000). "La traduction comme source pour l'étude d'anciens états de langue." Le Moyen Français 44-45, 243-264.

Guichard-Tesson, Françoise (1990). "Le métier de traducteur et de commentateur au $\mathrm{XIV}^{\mathrm{e}}$ siècle d'après Evrart de Conty." Le Moyen Français 24-25, 131-167.

Guichard-Tesson, Françoise (1993). "Le souci de la langue et du style au XIV siècle: l'autographe des Problèmes d'Evrart de Conty." Le Moyen Français $33,57-84$.

Kühner, Raphael \& Friedrich Holzweissig (21912). Ausführliche Grammatik der Lateinischen Sprache. I. Elementar-, Formen- und Wortlehre. Hannover. [Reprint 1994. Hannover: Hahn]

Louis, Pierre (Ed.) (1991-1994). Aristote. Problèmes. Texte établi et traduit. Collection des Universités de France. Paris: Les Belles Lettres.

Lusignan, Serge (1986). Parler vulgairement. Les intellectuels et la langue française aux XIII ${ }^{e}$ et XIV ${ }^{e}$ siècles. Etudes Médiévales. Montréal: Les Presses de l’Université de Montréal - Paris: J. Vrin.

Marx, Fridericus (Ed.) (1964) ('1923). Incerti auctoris De Ratione Dicendi ad C. Herennium lib. IV. Editionem stereotypam correctiorem cum addendis curavit Winfried Trillitzsch. (M. Tulli Ciceronis scripta quae manserunt omnia 1. Bibliotheca scriptorum Graecorum et Romanorum Teubneriana). Leipzig: Teubner.

Monfrin, Jacques (1964). "Humanisme et traductions au Moyen Age." L'humanisme médiéval dans les littératures romanes du XIII e au XIV e siècle... (Actes et Colloques 3). Paris: Klincksieck, 217-262. 
OLD = Oxford Latin Dictionary (1968-1982). Oxford: Clarendon.

Rey, Alain (Dir.) (1992). Dictionnaire historique de la langue française. Paris: Dictionnaires Le Robert.

Smets, An (2000). "Les traductions en moyen français des traités cynégétiques latins: le cas du De Falconibus d'Albert le Grand." A. Paravicini-Bagliani \& B. van den Abeele (Eds) (2000). La chasse au Moyen Age: Société, traités, symboles. Micrologus Library 5. Firenze: Sismel, Edizioni del Galluzzo, 71-85.

Stroebel, E. (Ed.) (1965) ('1915). Rhetorici libri duo qui vocantur De Inventione. Editio stereotypa editionis prioris (MCMXV). (M. Tulli Ciceronis scripta quae manserunt omnia 2. Bibliotheca scriptorum Graecorum et Romanorum Teubneriana). Stuttgart: Teubner.

TL: voir Tobler \& Lommatzsch $1915 \mathrm{ss}$

Tobler, A. \& E. Lommatzsch. (1915ss.). Altfranzösisches Wörterbuch. Adolf Toblers nachgelassene Materialien bearbeitet und herausgegeben von Ehrhard Lommatzsch. Berlin/Wiesbaden: Fr. Steiner.

Van Hoecke, Willy (Ed.) (s.d.) "La Rettorique de Marc Tulles Cyceron". La traduction par Jean d'Antioche (1282) du "De Inventione" de Cicéron et de la "Rhetorica ad Herennium" éditée d'après le manuscrit unique. Louvain.

Van Hoecke, Willy. (à par.). "Problèmes de traducteurs: la négation du latin au français". Revue romane.

Wartburg, Walter von (1928ss.). Französisches Etymologisches Wörterbuch. Eine Darstellung des galloromanischen Sprachschatzes. Bonn: Klopp/Bâle: Zbinden.

${ }^{1}$ Pour plus de détails, nous renvoyons à Goyens \& Van Hoecke (2000:250ss.).

${ }^{2}$ Ces chiffres sont basés sur les 2500 premiers syntagmes nominaux du corpus de base, et plus précisément de la traduction par Jean d'Antioche en ancien français et ses correspondants dans la traduction en français moderne de Bornecque (1932). Pour plus de détails, nous renvoyons à Goyens (1994:224).

${ }^{3}$ Pour plus de détails, voir Carlier \& Goyens (1998).

${ }^{4}$ Voir par exemple l'étude de Van Hoecke (à par.)

${ }^{5}$ K.U.Leuven, Onderzoeksfonds, projet $n^{\circ}$ OT/01/06.

${ }^{6}$ L'édition du De Historia Animalium est préparée par F. Bossier et P. Beullens. Les livres I-V ont été publiés en 2000 (cf. Beullens \& Bossier éds 2000); les livres VI-X sont prêts pour publication.

${ }^{7}$ L'Aristoteles Latinus est un projet international d'éditions, coordonné par l'Union Académique Internationale, dont le but est d'éditer toutes les traductions médiévales, du grec au latin, des oeuvres d'Aristote. Ce centre est hébergé dans l'Institut Supérieur de Philosophie de la K.U. Leuven. Les collègues de ce Centre collaborant au projet sont Jozef Brams, Pieter De Leemans et Maria Fredriksson.

${ }^{8}$ Pour plus de détails concernant ces textes, voir Smets (2000).

${ }^{9}$ Le texte grec d'Aristote a été édité et traduit encore récemment par Louis (éd. 1991-1994).

${ }^{10}$ Mis à part le texte grec original, les textes qui font partie de ce corpus n'ont pas encore été édités, ou seulement en partie. La traduction en latin de Barthélémy de Messine n'a pas encore été éditée, mis à part quelques fragments qui ont été édités de façon semi-critique. Pieter De Leemans s'occupe de l'édition de cette traduction. Le texte est conservé dans plus de 50 mss., dont le plus important semble être PATAVINUS, Bibl. Antoniana, Scaff. XVII, 370 (14e s.). Le commentaire de Pierre d'Abano n'a pas encore été édité: sa tradition manuscrite n'est d'ailleurs pas encore bien connue. Notons toutefois que 4 mss conservant la traduction de Barthélémy de 
Messine présentent conjointement le commentaire de Pierre d'Abano. Enfin, la traduction française d'Evrart de Conty n'a pas encore été éditée non plus. Elle a déjà été étudiée par Guichard-Tesson (1990, 1993); l'édition est préparée par Michèle Goyens et Françoise Guichard-Tesson. Pour de plus amples informations sur Evrart de Conty, nous renvoyons aux études de Guichard-Tesson (1990 et 1993).

${ }^{11}$ Goyens \& De Leemans (à par.) ont comparé les stratégies de traduction des deux traducteurs, et l'apport particulier du commentateur. Goyens (à par. a, b) a étudié le lexique relatif à la mélancolie et celui des plantes dans la traduction d'Evrart de Conty.

${ }^{12}$ Nous citons le texte d'Evrart de Conty d'après le manuscrit Paris, BNF, fr. 2428124282, communément admis comme étant un autographe.

${ }^{13}$ GOD (IX: 130) cite l'extrait d'Evrart de Conty en premier lieu, et ajoute des exemples plus tardifs, tirés de la Mer des hystoires (t II, fol. 3d) de Pierre le Rouge (15e s.), et du Jardin de santé (fin 15e s.). 